\title{
Prosodic and pragmatic values of discourse particles in French
}

\author{
Lou Lee ${ }^{1}$, Katarina Bartkova ${ }^{1}$, Mathilde Dargnat ${ }^{1}$, Denis Jouvet ${ }^{2}$ \\ ${ }^{1}$ Université de Lorraine, CNRS, ATILF, F-54000 Nancy, France \\ ${ }^{2}$ Universite de Lorraine, CNRS, Inria, LORIA, F-54000 Nancy, France \\ https://doi.org/10.36505/ExLing-2018/09/0018/000351
}

\begin{abstract}
This paper analyses prosodic properties of three discourse particles (DP) in French: 'alors' ('so'), 'bon' ('well') and 'donc' ('thus'), according to their different pragmatic functions. DP occurrences of these words extracted from a large speech database have been annotated manually with pragmatic labels. For each DP, prosodic characteristics of occurrences of each pragmatic function (conclusive, introductive, etc.) are automatically extracted. Then, for each DP and each pragmatic function, the most frequent F0 forms are retained as the representative forms. Results show that a pragmatic function, common to several discourse particles, gives rise to a uniform prosodic marking, and this lead to suppose that such DPs are most of the time commutable.
\end{abstract}

Key words: discourse particles, prosody, pragmatics, computational linguistics

\section{Introduction}

Discourse is defined as intrinsically interactional, or dialogic (Bakhtine 1978; Benveniste 1958; a.o.). Spoken language contains discourse particles (DPs), that are cues for discourse or interaction interpretation (Aijmer 2013; Dostie 2004).

We investigate here whether prosodic properties of DPs in French provide information that is related to their various pragmatic values they convey ('introduction', 'conclusion', 'comment', 'emotional state', etc.). Although there are many studies on DPs, very few are dealing with their prosodic correlates.

In this paper, a systematic study is carried out on the prosodic specificities of three French DPs ('alors', 'donc' and 'bon') with respect to their pragmatic functions. About 1000 occurrences of the three words ('alors', 'donc', 'bon') have been randomly extracted from several French speech corpora. Each occurrence has been manually annotated, first as DP / non-DP (Bartkova 2016), and with pragmatic labels when DP.

\section{Methodology and data base}

The main goal in this study is to evaluate how different pragmatic functions of the three DPs are defined prosodically. Hence, F0 patterns

ExLing 2018: Proceedings of 9th Tutorial and Research Workshop on Experimental Linguistics, 28-30 August, Paris, Frannce 
of the pragmatic functions are extracted and their forms are studied jointly with the position of the DP occurrences inside the prosodic groups.

DPs are extracted from more than 100 hours of French speech corpora of various degree of spontaneity, coming from the ESTER2 evaluation campaign (Galliano 2009) and the ORFEO project (ORFEO).

All the data is segmented automatically into phonemes and words, using speech-text forced alignment. The automatic detection of prosodic groups is based on F0 slope values, pitch level and vowel duration. F0 values are normalized according to the speaker's pitch range.

\section{DPs' prosodic articulation}

F0 patterns reflect prosodic articulations between DPs and their immediate contexts. The DPs prosodic articulations are studied with respect to their pragmatic functions. For this, the syllable nuclei under consideration are the last syllable of the left context (' $w-1$ '), the last syllable nuclei of the DP, and the first syllable of the right context ('w+1').

The movements of F0 between ' $w-1$ ' and the DP, and between the DP and ' $w+1$ ', are classified into 3 classes according to the F0 slope directions: falling, rising and plateau. Figure 1 gives examples of F0 patterns.

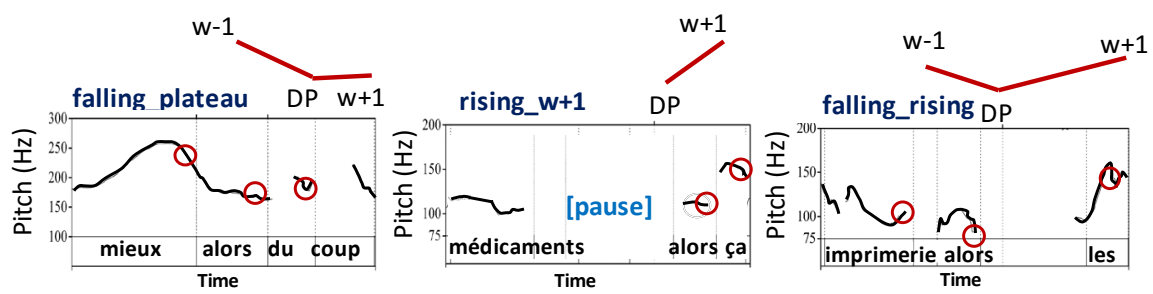

Figure 1: Example of the some frequent F0 patterns.

\section{F0 pattern modelling}

Typical F0 patterns per pragmatic function are obtained using a vector quantization procedure. The representative $\mathrm{F} 0$ patterns correspond to the centroid of the class. Some F0 patterns are very similar from one function to another; yet, some others reflect prosodic differences among the pragmatic functions. For the DP 'alors', for example, Figure 2 shows a 'falling-plateau' pattern with lower values for 'conclusion' function than for 'introduction' or 're-introduction' functions. 


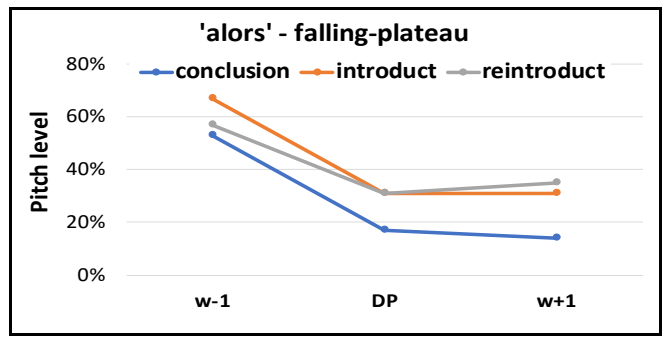

Figure 2: Comparison of stylized F0 patterns for 'alors'.

\section{Representative patterns of pragmatic functions}

Table 1 displays the most representative F0 pattern(s) for each pragmatic value of each DP. It is observed that the 'falling' pattern followed by either a 'plateau' or a 'rising' slope, is favoured by 'conclusion', 'confirmation' and 'incident pragmatic functions. 'Conclusion' and 'confirmation' functions express a 'look-back' semantic action of "finality" marked prosodically by a 'falling-rising' pattern highlighting a strong semantic break. 'Incident' represented mainly by a 'falling-plateau' pattern introduces a parenthetical comment. 'Introduction' and 'reintroduction' functions have a 'look-ahead' semantic action, prosodically confirmed by a 'rising', and a 'rising-plateau' patterns. In fact, the prosodic patterns of the same pragmatic values are also very similar, and no major differences exist among the 'falling-rising' patterns of the DPs. Therefore, one can suggest that these DPs in this pragmatic function are commutable and their only distinctive mark is not lexical but a prosodic one.

Table 1: Representative F0 patterns.

\begin{tabular}{|l|l|l|}
\hline DP & Pragmatic value & Representative F0 pattern \\
\hline \multirow{4}{*}{ 'alors' } & Conclusion & falling-rising \& falling-plateau \\
\cline { 2 - 3 } & Introduction & rising \& rising-plateau \\
\cline { 2 - 3 } & Reintroduction & falling-plateau \& plateau \\
\hline \multirow{3}{*}{ donc' } & Conclusion & falling-plateau \& plateau \\
\cline { 2 - 3 } & Reintroduction & rising-plateau \& plateau \\
\cline { 2 - 3 } & Addition & falling-plateau \& plateau \\
\hline \multirow{4}{*}{ bon' } & Conclusion & falling-rising \& falling-plateau \\
\cline { 2 - 3 } & Interruption & plateau \\
\cline { 2 - 3 } & Confirmation & falling-rising \& plateau \\
\cline { 2 - 3 } & Incident & falling-plateau \\
\hline
\end{tabular}




\section{Position in prosodic groups (PG)}

Though semantically related to sentences, DPs present a relative syntactic and prosodic independence in the sentence. Most of the time, DPs occur alone in prosodic groups. That is, even in absence of pauses, DPs are prosodically separated from their left and right contexts. 'Alors' is found in single word PGs $82 \%$ when 'introduction' and $90 \%$ when 'conclusion'. 'Bon' is encountered alone in PGs from $77 \%$ to $89 \%$ of the cases depending on pragmatic functions. 'Donc' occurs in single word GPs in more than $80 \%$ of cases.

\section{Conclusion}

This prosodic analysis show that particle pragmatic functions have prosodic specificities and these prosodic marks are related more to the pragmatic function than to the lexical content of the words studied here. An F0 modelling procedure using F0 levels allowed extracting the most prominent F0 patterns for each pragmatic function. In fact, for a given pragmatic value, the prosodic patterns are very similar. Therefore, it can be supposed that DPs having the same pragmatic function can be interchangeable in the speech chain, and further work is on-going to investigate this hypothesis.

\section{Acknowledgements}

This work has been partially supported by the CPER LCHN (Contrat Plan Etat Région "Langues, Connaissances et Humanités Numériques").

\section{References}

Bakhtine M. 1978. Esthétique et théorie du roman. Paris: Gallimard.

Benveniste E. 1958. De la subjectivité dans le langage, in Problèmes de linguistique générale, tome 1, 258-266. Paris: Gallimard.

Aijmer K. 2013. Understanding Pragmatic Markers: A Variational Pragmatic Approach. Edinburgh: Edinburgh University Press.

Dostie G. 2004. Pragmaticalisation et marqueurs discursifs. Analyse sémantique et traitement lexicographique. Bruxelles: DeBoeck/Duculot.

Bartkova K., Bastien A., Dargnat M. 2016. How to be a Discourse Particle?, Proceedings of Speech Prosody 2016, 858-863, Barnes, J., Brugos, A., ShattuckHufnagel, S., Veilleux, N. (Eds), Boston, USA.

Galliano S., Gravier G., Chaubard L. 2009. The ESTER 2 evaluation campaign for rich transcription of French broadcasts, Proc. Interspeech 2009, 2583-2586, 10th Annual Conf. of the Int. Speech Communication Association, Brighton.

ORFEO project: http://www.projet-orfeo.fr/ 